\title{
Estimating pre-harvest density, adult sex ratio and fecundity of white-tailed deer using wildlife cameras
}

\author{
Jon Brommer ${ }^{1}$, Jenni Poutanen ${ }^{1}$, Jyrki Pusenius², and Mikael Wikström \\ ${ }^{1}$ University of Turku \\ ${ }^{2}$ Natural Resources Institute Finland \\ ${ }^{3}$ Finnish Wildlife Agency
}

April 12, 2021

\begin{abstract}
Adult sex ratio and fecundity are key population parameters in sustainable wildlife management, but inferring these requires estimates of the density of at least three age/sex classes of the population (male and female adults and juveniles). We used an array of 36 wildlife camera traps during 2-3 weeks in autumn prior to harvest during two consecutive years, and recorded white-tailed deer adult males, adult females and fawns from the pictures. Simultaneously, we collected fecal DNA (fDNA) from $9220 \mathrm{mx} 20 \mathrm{~m}$ plots placed in 23 clusters of four plots between the camera traps. We identified individuals from fDNA samples with microsatellite markers and estimated the total sex ratio and population density using Spatial Capture Recapture (SCR). The fDNA-SCR analysis concluded equal sex ratio in the first year and female bias in the second year, and no difference in space use between sexes (fawns and adults combined). Camera information was analyzed in a Spatial Capture (SC) framework assuming an informative prior for animals' space use, either (1) as estimated by fDNA-SCR (same for all age/sex classes), (2) as assumed from the literature (space use of adult males larger than adult females and fawns), (3) by inferring adult male space use from individually-identified males from the camera pictures. These various SC approaches produced plausible inferences on fecundity, but also inferred total density to be lower than the estimate provided by fDNA-SCR in one of the study years. SC approaches where adult male and female were allowed to differ in their space use suggested the population had a female-biased adult sex ratio. In conclusion, SC approaches allowed estimating the pre-harvest population parameters of interest and provided conservative density estimates.
\end{abstract}

\section{Hosted file}

Estimating pre-harvest density Brommer et al.pdf available at https://authorea.com/users/ 407290/articles/517635-estimating-pre-harvest-density-adult-sex-ratio-and-fecundity-ofwhite-tailed-deer-using-wildlife-cameras 